\title{
28 Research Suare \\ Spectrum of biopsy renal disease in central China: a nine-year retrospective study of 1929 cases
}

Jianjiang Zhang ( $\sim 15936269856 @ 163 . c o m$ )

The First Affiliated Hospital of Zhengzhou University

Jin Zhang

The First Affiliated Hospital of Zhengzhou University

\section{Huiqin Zeng}

The First Affiliated Hospital of Zhengzhou University

Peipei Shi

The First Affiliated Hospital of Zhengzhou University

Wanyu Jia

The First Affiliated Hospital of Zhengzhou University

Zhen Liu

The First Affiliated Hospital of Zhengzhou University

\section{Research Article}

Keywords:

Posted Date: February 14th, 2022

DOI: https://doi.org/10.21203/rs.3.rs-1289039/v1

License: (c) (i) This work is licensed under a Creative Commons Attribution 4.0 International License.

Read Full License 


\section{Abstract}

Objective To investigate the renal pathology and transitions of children who received a renal biopsy at the First Affiliated Hospital of Zhengzhou University between 2012 and 2020.

Methods The clinical and pathological data of children with a renal biopsy were analyzed retrospectively. The changes in the distribution of pathological types were examined in age groups of 3 years $(-1,-3,-6$, $-12,-16$ years) and gender.

Results 1928 children with renal biopsies were included in the analysis. The mean age of the children was $8.18 \pm$ SD 3.40 years and the ratio of males to females was 1.71 . The main pathological types of primary glomerular disease were minimal change disease (MCD) and IgA nephropathy, while the main pathological types of secondary glomerulonephritis were lupus nephritis and Henoch-Schonlein purpura (HSPN). The male to female ratio and age distribution between lupus nephritis and HSP were significantly different.

\section{Introduction}

Kidney disease is common in pediatric patients and can be divided into primary, secondary, and hereditary kidney diseases. Renal biopsy is an important diagnostic tool used to obtain the diagnosis of kidney pathology. Although laboratory and radiological techniques help to diagnose kidney disease, renal biopsy remains the most important procedure used for the management of renal disease in children.

Kidney disease in children varies across regions, races, genders, ages, and eras. Differences in the indication for a kidney biopsy between regions and generations may affect the spectrum of renal biopsy disease $^{1-5}$. In 1996, the Subspecialty Group of Nephrology of the Society of Pediatrics in the Chinese Medical Association analyzed the pathology of 2315 renal biopsies from 20 clinical units and reported the pathology of kidney biopsies in children in several areas of China.

The current study retrospectively analyzed the pathological data of renal biopsies of children collected since 2012 at the First Affiliated Hospital of Zhengzhou University. The data was used to clarify the prevalence and changes in renal disease over the last 9 years.

\section{Patients And Methods}

\section{Inclusion and exclusion criteria}

Cases of renal biopsies performed at the Department of Pediatric Nephrology, The First Affiliated Hospital of Zhengzhou University from 2012 to 2020 were investigated. All the children were $\leq 16$ years of age at the time of the renal biopsy. Cases with less than 5 glomeruli observed by light microscopy and no obvious lesions in the renal biopsy were excluded. 


\section{Indications for kidney biopsy}

The children underwent a renal biopsy for the following conditions. Refractory nephrotic syndrome, including frequent relapses, hormone dependence and hormone resistance; persistent proteinuria and/or glomerulogenic hematuria; acute glomerulonephritis starting with hypocomplementemia for more than 8 weeks; acute nephritis, hereditary nephritis, and secondary glomerular disease; and acute and chronic renal insufficiency of unknown origin.

\section{Renal Biopsy Methods}

Before the renal biopsy, the child's family was informed of the purpose and indications of the procedure and possible complications, followed by signed consent being obtained. The child was placed in a prone position during the puncture, with the puncture point positioned under the right kidney. Under the guidance of ultrasound positioning, a fully automatic puncture biopsy gun was held and inserted vertically into the renal cortex, the trigger of the gun pressed, followed by the gun being quickly withdrawn to successfully obtain kidney tissue.

\section{Renal histopathology}

The kidney tissue obtained was divided into three parts for light microscopy, fluorescence, and electron microscopy.

Light microscopy: The light microscopy specimens were placed in $4 \%$ neutral formaldehyde fixative (also known as $10 \%$ formalin). Paraffin-embedded sections were then prepared and stained with HE, PASM, or PASM+Masson stains. After staining, the pathological changes of glomeruli, tubules, interstitium, and renal vessels were observed under a light microscope.

Immunofluorescence: IgG, IgA, IgM, C3, Clq, and FRA fluorescence staining were routinely performed on frozen sections and sometimes antigen testing was performed as needed, such as detection of hepatitis $B$ virus antigen ( $\mathrm{HBsAg}, \mathrm{HBcAg}$ ) and $\mathrm{k}$ and $\lambda$ light chain proteins. The intensity, type, location, and shape of the immune complexes observed by immunofluorescence microscopy were recorded. Type IV collagen a1, a3, and a5 assays were added after 2018.

Electron microscopy: Fresh kidney tissues were fixed with $2.5 \%$ cold glutaraldehyde and $2 \%$ osmium tetroxide, then dehydrated with ethyl alcohol or acetone and embedded in epoxy resin Epon812. Ultrathin sections were prepared and stained with uranyl acetate and lead citrate and observed by electron microscopically at the First Hospital of Peking University and the Department of Pathology of our hospital.

\section{Grouping methods}


To examine changes in the disease spectrum over time the cases were divided into three time periods: 2012-2014, 2015-2017, and 2018-2020. To determine the distribution of diseases in each age group, the cases were grouped by age as $\leq 1, \sim 3, \sim 6, \sim 12$, and $\sim 16$ years.

\section{Statistical methods}

The numbers of patients in each group were expressed as percentages. Differences in the rates of the diseases were analyzed using the chi-square test. $P$-values $<0.05$ indicated that the differences were statistically significant. SPSS 25.0 software was used for the statistical analyses.

\section{Results}

\section{General information}

Based on the inclusion and exclusion criteria a total of 1928 cases were included in the analysis. Of the 1928 cases, 1219 (63.23\%) cases were males and 709 (36.77\%) were females. The average age of the study cohort was $8.2 \pm 3.4$ years and the ratio of males to females was 1.71 . The incidence in all kinds of renal diseases in children in this study accounted for $57.78 \%$ (1114 cases) was highest in those attending school (6 12 years) (Fig. 1a,b). In terms of age proportion, children with primary and hereditary kidney diseases were younger than those with secondary kidney diseases.

\section{Composition of the kidney disease type}

Of the 1928 children with a renal biopsy, 1074 had primary nephropathy, 758 had secondary nephropathy, and 96 had hereditary nephropathy. Primary glomerular disease consists with glomerular minor lesion/minimal change disease(MCD), mesangial proliferative glomerulonephritis(MsPGN), IgM nephropathy(IgMN), IgA nephropathy(IgAN), membranous nephropathy(MN), focal segmental glomerulosclerosis(FSGS), endocapillary proliferative glomerulonephritis(EPGN), focal proliferative glomerulonephritis(FPGN), membranous proliferative glomerulonephritis(MPGN), C3 glomerulonephritis(C3GN), interstitial nephropathy(IN) and others, such as C1q nephropathy (1 case), endotheliosis(1 case), dense deposit disease(DDD, 2 cases), focal sclerotic glomerulonephritis(2 cases), crescentic glomerulonephritis(CGN, 1 case), post-infectious glomerulonephritis(PIGN, 1 case), diffuse mesangial hyperplasia and sclerosis(1case), ANCA-associated vasculitis(AAV, 1 case), ischemic kidney injury(1 case). Secondary glomerular disease consists with lupus nephritis(LN), Henoch-Schonlein purpura nephritis(HSPN), hemolytic uremic syndrome(HUS), HBV-associated glomerulonephritis(HBVGN). Hereditary glomerular disease includes Thin basement membrane disease(TBMD), Alport syndrome(AS) and basement membrane disease.

The most common types of pathology in primary kidney disease were MCD and IgAN, accounting for $40.26 \%$ and $34.86 \%$ of cases, respectively. Secondary nephropathy was dominated by HSPN and LN, 
accounting for $78.52 \%$ and $17.52 \%$ of cases, respectively. Hereditary nephropathy was dominated by Alport syndrome (59.38\%) and thin basement membrane nephropathy (32.29\%).

\section{Table 1}

The gender distributions of primary glomerular disease according to pathological findings. [n (\%)].

\begin{tabular}{|lllll|}
\hline Primary glomerular disease & All & Male & Female & $p$ \\
& $\mathrm{n}=1074$ & $\mathrm{n}=733$ & $\mathrm{n}=341$ & \\
\hline MCD & $432(40.2)$ & $309(42.2)$ & $123(36.1)$ & 0.058 \\
\hline MsPGN & $36(3.4)$ & $28(3.8)$ & $8(2.3)$ & 0.212 \\
\hline IgMN & $20(1.9)$ & $12(1.6)$ & $8(2.3)$ & 0.424 \\
\hline IgAN & $374(34.8)$ & $257(35.1)$ & $117(34.3)$ & 0.864 \\
\hline MN & $33(3.1)$ & $22(3.0)$ & $11(3.2)$ & 0.843 \\
\hline FSGS & $80(7.4)$ & $51(7.0)$ & $29(8.5)$ & 0.369 \\
\hline EPGN* & $34(3.2)$ & $17(2.3)$ & $17(5.0)$ & 0.020 \\
\hline FPGN* & $24(2.2)$ & $11(1.5)$ & $13(3.8)$ & 0.017 \\
\hline MPGN & $7(0.7)$ & $3(0.4)$ & $4(1.2)$ & 0.298 \\
\hline C3GN & $13(1.2)$ & $7(1.0)$ & $6(1.8)$ & 0.411 \\
\hline IN & $10(0.9)$ & $6(0.8)$ & $4(1.2)$ & 0.824 \\
\hline Others & $11(1.0)$ & $10(1.4)$ & $1(0.3)$ & 0.195 \\
\hline
\end{tabular}

\section{Table 2}

The age distributions of primary glomerular disease according to pathological findings. [n (\%)]. 


\begin{tabular}{|llllllll|}
\hline Primary glomerular & $\sim 1$ & $\sim 3$ & $\sim 6$ & $\sim 12$ & $\sim 18$ & $\chi 2$ & $p$ \\
\hline MCD & $\mathrm{n}=21$ & $\mathrm{n}=134$ & $\mathrm{n}=279$ & $\mathrm{n}=560$ & $\mathrm{n}=80$ & & \\
\hline MsPGN & $9(42.9)$ & $84(62.7)$ & $154(55.2)$ & $162(28.9)$ & $23(28.8)$ & 88.3 & 0.000 \\
\hline IgMN & $1(4.8)$ & $13(9.7)$ & $11(2.9)$ & $10(1.8)$ & $1(1.2)$ & 18.4 & 0.001 \\
\hline IgAN & $1(4.8)$ & $8(6.0)$ & $3(1.0)$ & $5(0.9)$ & $3(3.8)$ & 16.2 & 0.001 \\
\hline MN & $1(4.8)$ & $2(1.5)$ & $62(22.2)$ & $284(50.7)$ & $25((31.3)$ & 156.2 & 0.000 \\
\hline FSGS & 0 & $4(3.0)$ & $10(3.6)$ & $11(2.0)$ & $8(10.0)$ & 11.9 & 0.012 \\
\hline EPGN & $6(28.6)$ & $14(10.4)$ & $18(6.5)$ & $34(6.1)$ & $8(10.0)$ & 18.0 & 0.002 \\
\hline FPGN & $1(4.8)$ & $1(0.7)$ & $6(2.2)$ & $24(4.3)$ & $2(2.5)$ & 6.2 & 0.152 \\
\hline MPGN & $2(1.0)$ & $3(2.2)$ & $9(3.2)$ & $9(1.6)$ & $1(1.3)$ & 6.7 & 0.113 \\
\hline C3GN & 0 & $2(1.5)$ & $1(0.4)$ & $2(0.4)$ & $2(2.5)$ & 6.6 & 0.095 \\
\hline IN & 0 & $1(0.7)$ & $3(1.1)$ & $7(1.3)$ & $2(2.5)$ & 1.7 & 0.758 \\
\hline Others & 0 & 0 & $1(0.4)$ & $7(1.3)$ & $2(2.5)$ & 4.5 & 0.273 \\
\hline
\end{tabular}

Further analysis by gender showed statistically significant differences in the gender distribution of EPGN and FPGN in primary kidney disease $(p=0.020$ and 0.017$)$. Analysis also showed that the prevalence of EPGN and FPGN was higher in females than in males. The difference in the number of male patients versus female patients for the other pathological types of primary nephropathy was not significantly different (Fig 2a, Table 1).

Of the primary glomerular diseases, the number of MCD, MsPGN, IgMN, IgAN, MN, FSGS showed statistically significant age-related differences $(p=0.000,0.001,0.001,0.000,0.012,0.002)$. However, there were no statistically significant differences in the distribution of the pathological types of the other primary glomerular disease grouped according to age. Children with MCD, IgAN, MN, and FSGS were mainly preschool- and school-age. Children with IgMN were predominantly early childhood age, while MPGN tended to be more distributed in early childhood, preschool, and school ages (Fig 2b, Table 2).

\section{Table 3}

The gender distributions of secondary glomerular disease according to pathological findings. [n (\%)]. 


\begin{tabular}{|lllll|}
\hline Secondary glomerular disease & All & Male & Female & $p$ \\
& $\mathrm{n}=758$ & $\mathrm{n}=423$ & $\mathrm{n}=335$ & \\
\hline LN** & $133(17.5)$ & $16(3.8)$ & $117(34.9)$ & 0.000 \\
\hline HSPN** & $596(78.6)$ & $393(92.9)$ & $203(60.6)$ & 0.000 \\
\hline HUS & $19(2.5)$ & $9(2.1)$ & $10(3.0)$ & 0.457 \\
\hline HBV-GN & $10(1.3)$ & $5(1.2)$ & $5(1.5)$ & 0.963 \\
\hline
\end{tabular}

\section{Table 4}

The age distributions of secondary glomerular disease according to pathological findings. [n (\%)].

\begin{tabular}{|llllllll|}
\hline Secondary glomerular disease & $\sim 1$ & $\sim 3$ & $\sim 6$ & $\sim 12$ & $\sim 18$ & $\chi 2$ & $p$ \\
& $\mathrm{n}=1$ & $\mathrm{n}=4$ & $\mathrm{n}=122$ & $\mathrm{n}=510$ & $\mathrm{n}=121$ & & \\
\hline LN** & 0 & 0 & $2(1.6)$ & $89(17.5)$ & $42(34.7)$ & 52.4 & 0.000 \\
\hline HSPN** & 0 & $3(75)$ & $113(92.6)$ & $403(79)$ & $77(63.6)$ & 34.8 & 0.000 \\
\hline HUS** & 1 & 0 & $7(5.7)$ & $11(2.2)$ & 0 & 18.0 & 0.002 \\
\hline HBV-GN* & 0 & $1(25)$ & 0 & $7(1.4)$ & $2(1.7)$ & 11.0 & 0.044 \\
\hline
\end{tabular}

Among the secondary nephropathies, the difference in gender distribution for LN and HSPN was significantly different ( $p \bigotimes 0.01$ ). Data analysis also showed that the number of girls with LN was higher than for boys, while the opposite trend was observed for HSPN. There was no significant difference in gender distribution for the HUS and HBV-GN (Fig 3a, Table 3).

The differences in the age distribution of children with secondary glomerular disease were all statistically significant, with the data showing secondary nephropathy occurred mainly in school-age children (Fig 3b, Table 4).

\section{Table 5}

The gender distributions of hereditary glomerular disease according to pathological findings. [n (\%)].

\begin{tabular}{|lllll|}
\hline Hereditary glomerular disease & All & Male & Female & $p$ \\
& $\mathrm{n}=96$ & $\mathrm{n}=63$ & $\mathrm{n}=33$ & \\
\hline TBMD & $31(32.3)$ & $13(20.6)$ & $18(54.5)$ & 0.001 \\
\hline AS & $57(59.4)$ & $46(73.0)$ & $11(33.3)$ & 0.000 \\
\hline Basement membrane disease & $8(8.3)$ & $4(6.3)$ & $4(12.1)$ & 0.560 \\
\hline
\end{tabular}




\section{Table 6}

The age distributions of hereditary glomerular disease according to pathological findings. [n (\%)].

\begin{tabular}{|llllllll|}
\hline Hereditary glomerular disease & $\sim 1$ & $\sim 3$ & $\sim 6$ & $\sim 12$ & $\sim 18$ & x2 & $\mathrm{p}$ \\
& $\mathrm{n}=1$ & $\mathrm{n}=16$ & $\mathrm{n}=31$ & $\mathrm{n}=44$ & $\mathrm{n}=4$ & & \\
\hline TBMD & 0 & $1(6.3)$ & $8(25.8)$ & $19(43.2)$ & $3(75)$ & 11.8 & 0.010 \\
\hline AS & 1 & $14(87.5)$ & $21(67.7)$ & $20(45.5)$ & $1(25)$ & 12.3 & 0.007 \\
\hline Basement membrane disease & 0 & $1(6.3)$ & $2(6.5)$ & $5(11.3)$ & 0 & 1.6 & 0.925 \\
\hline
\end{tabular}

The hereditary glomerular diseases showed significant differences in gender distribution for TBMD and AS ( $p \llbracket 0.01)$. TBMD was more prevalent in girls than in boys, whereas AS was more prevalent in boys than in girls (Fig 4a, Table 5).

Of the hereditary glomerular diseases, the differences in distribution of TBMD and AS were statistically significant across the age groups $(p=0.010,0.007)$, with both diseases being more prevalent in preschool and school-age children (Fig 4b, Table6).

\section{Composition of pathological types by time group}

The data collected in this study were divided into the first three years, the middle three years, and the last three years.

Among the primary glomerular diseases, the differences in the temporal distribution of MCD, MsPGN, IgAN and C3GN were statistically significant $(p=0.037,0.001,0.000,0.001)$. The number of cases of MCD and MsPGN has decreased gradually since 2012, whereas the number of cases of IgAN and C3GN has increased gradually over the same time period. The differences in the temporal distribution of the other types of primary glomerular disease were not statistically significant (Fig 5a, b).

For the secondary glomerular diseases, there was a significant difference in the temporal distribution of LN and HSPN ( $p=0.000,0.001)$, whereas there was no significant difference in the other types of diseases. The number of LN cases showed an upward trend, while cases of HSPN gradually decreased (Fig5c, d).

For the hereditary glomerular diseases, differences in the time distribution of basement membrane lesions were statistically significant $₫ p \otimes 0.05$ ), while cases of Alport syndrome and thin basement membrane nephropathy showed no significant time-related changes (Fig5e, f).

\section{Discussion}


This study collated the basic information and pathological types of renal disease in children who underwent a renal biopsy at the Department of Pediatrics of the First Affiliated Hospital of Zhengzhou University between 2012 and 2020. This study included analysis of the gender and age distribution of different kidney diseases and examined the changes in each type of kidney disease over 9 years. In the past 9 years, the number of kidney biopsies performed at our center has increased every year as a result of an increase in the number of beds, the development of kidney biopsy technology, and the economic situation of the patients' families.

\section{Composition of pathological types}

The study showed that primary glomerular diseases were the most common kidney disease in the children in our study, followed by secondary glomerular disease and hereditary nephropathy. These results are consistent with those in domestic and most international studies.

The most common pathological types of primary nephropathy were MCD and IgAN, which accounted for $40.2 \%$ and $34.8 \%$ of primary nephropathies, respectively. This differs from a previous study that reported the main pathological type of primary kidney disease was MsPGN. ${ }^{1}$ This inconsistency between the studies may be a consequence of the primary etiology of the kidney biopsy patients. In recent years, the main cause of primary kidney disease in our center was nephrotic syndrome. ${ }^{12}$ The pathological manifestations of the nephrotic syndrome were predominantly MCD and IgAN. Kidney biopsies of children with different etiologies may lead to changes in the spectrum of renal pathology observed. With the advances in renal pathology and pathology, many causes of MsPGN can be identified and classified into various types of secondary glomerulonephritis. Primary MsPGN is relatively uncommon, with the majority of domestic studies including Taiwan studies having shown that MCD is the main pathological type of primary glomerular disease ${ }^{5}$. Some studies in Western Asia, including Jordan, and East Asia including Korea, are more consistent with the findings of domestic studies ${ }^{6,11}$. A study in Egypt also confirmed this result ${ }^{10}$. However, a study in India showed that the most common primary glomerular disease in that country was FPGN and $\operatorname{IgMN}^{4}$. Studies in European countries including Italy and Croatia have confirmed that the main pathological types of primary glomerular disease are $\lg A N$ and $M C D^{7,16}$. This suggests that the disease spectrum of primary glomerular disease varies between countries, possibly related to factors such as region and race.

HSPN accounted for the highest percentage of secondary kidney disease at $78.6 \%$, followed by $L N$ at $17.5 \%$. HUS and HBV-GN were less frequent. The etiological diagnosis of secondary renal disease differed markedly between children and adults, with HSPN the predominate disease in children and LV the most common disease in adults. This is consistent with previous national and international descriptive statistics that the disease spectrum of secondary kidney disease in children is unique. $4,5,7,8$

The main pathological types of hereditary renal disease were Alport syndrome (59.4\%) and thin basement membrane nephropathy (32.3\%). 


\section{Gender}

Consistent with most national and international studies, the number of children with kidney disease was higher in males than in females, with the ratio of male to female being 1.71. The male to female ratio of renal biopsies in children reported in previous studies ranges from 1.1 to 1.7 with only an Egyptian study reporting an inverse ratio of 0.85 . This finding may be related to ethnic differences and different types of disease, and inclusion criteria for renal biopsies. ${ }^{2,4,5,8,9,10,11}$ For all types of kidney disease, the proportion of males with the disease was higher than in females, except for LN and TBMD. However, the predominance of males over females was statistically significant only for HSPN and AS. The prevalence of EPGN and FPGN was significantly higher ( $p \bigotimes 0.05)$ in females than in males. However, the gender differences in these two pathological types were less pronounced than for LN and TBMD ( $p \bigotimes 0.01)$.

The male to female ratio of children with $L N$ in this study was $1: 7.31 \rrbracket$ which contrasts to a previously reported overall male-to-female ratio of $1: 7$ to $9.5 .{ }^{13}$ In some studies, the proportion of male children with LN was significantly higher than that of adults. The prevalence of LN in male children is significantly higher than that observed in female children, especially in pre-puberty, with the ratio of males to females gradually approaching the adult level as age increased. ${ }^{3,5,14}$ As suggested by previous studies, this trend may be related to a close association between the effect of sex hormones on the development of LN. ${ }^{15,16}$ Estrogen and androgen levels are lower in children than in adults, which may explain the lower male-tofemale ratio in children with lupus nephritis compared to that in adults with the disease. SLE has a wide variety of clinical manifestations and can cause multiple systemic abnormalities with atypical presentations. Therefore, the value of renal biopsy in children with SLE should be emphasized in order that the pathological type is clarified in a timely manner and the activity of the lesion is determined accurately. This will lead to an early and correct diagnosis of the disease, development of a proper treatment plan, early treatment being initiated, and improved prognosis of the disease.

\section{Age}

Primary glomerular disease was the main cause of kidney disease in children in the age groups except for adolescent children. The prevalence of primary glomerular disease tended to decrease with age, whereas the prevalence of secondary glomerular disease tended to increase. Of the 177 cases aged $\leq 3$ years, primary glomerular disease accounted for $87.57 \%$ of cases, secondary glomerular disease $2.82 \%$, and hereditary glomerular disease $9.6 \%$. In the 432 preschool children (3-6 years), primary glomerular disease accounted for $64.58 \%$ of cases, secondary glomerular disease $28.24 \%$, and hereditary glomerular disease $7.18 \%$. Of the 1114 school-aged and 205 adolescent children, primary glomerular disease accounted for $50.27 \%$ and $39.02 \%$ of cases, secondary glomerular disease $45.78 \%$ and $59.02 \%$ of cases, and hereditary glomerular disease $3.95 \%$ and $1.95 \%$ of cases, respectively. Hereditary glomerulopathies were predominantly found in preschool and school age children. 
There were no obvious age-related differences in the pathological types of primary glomerular disease. However, a study in a large number of adult patients with nephropathy in China showed that the proportion of IgA nephropathy in primary glomerular disease gradually increased, while the proportion of MCD gradually decreased ${ }^{17}$. That study also showed that as age increased, the proportion of LN initially increased and then decreased, a trend which may be related to estrogen levels as mentioned above.

\section{Pathological changes}

In this study, the renal pathology over a nine-year period was divided into three regions: anterior, middle, and posterior.

In the primary glomerular diseases, the number of MCD and MSPGN gradually decreased, while the number of cases of IgAN and C3GN gradually increased. The decrease in the number of MCD may be related to recent advances in renal pathology, where some glomerular diseases presenting as MCD were diagnosed as IgAN, IgMN, or C3GN after immunofluorescence staining. ${ }^{17}$ The clinical symptoms of MsPGN present mainly as acute glomerulonephritis. In recent years, the prevalence of acute glomerulonephritis has decreased significantly compared to that measured in previous years due to improvements in living standards and health conditions of the general population. This has resulted in the number of cases of MSPGN also gradually decreasing. ${ }^{1,12}$

Among the secondary glomerular diseases, unlike those reported in several domestic children's hospitals, the present study showed a significant decrease in the prevalence of HSPN compared to that observed in recent years. ${ }^{18}$ It has been shown recently that the severity of pathological damage and prognosis of HSPN are closely related to the presence of massive proteinuria. Massive proteinuria is now also used as an important indicator for renal biopsy in clinical practice. This change may therefore be related to the choice of renal biopsy indicators in clinical practice.

There was no significant statistical difference in the spectrum of hereditary kidney diseases over the nine years of our study. Although the data showed significant changes in the spectrum of basement membrane disease, this trend might be related to the application of "basement membrane disease" in pathology in recent years. Although our data showed no significant difference in the number of cases of AS and TBMD over the nine-year study period, the proportion of these two diseases was higher than that observed in the previous three years. However, this finding may have been influenced by bias caused by the low number of cases of hereditary nephropathy.

\section{Conclusion}

This retrospective study analyzed renal biopsy pathological data from the Children's Nephrology Department of the First Affiliated Hospital of Zhengzhou University collected between 2012 and 2020. To a certain extent, this data represents the disease spectrum of children's kidney diseases in central China. 
The study showed that the main pathological types of primary glomerular disease were MCD and IgA nephropathy. There was no significant difference in the sex ratio and age distribution of primary glomerular disease. Over the nine years of the study, the prevalence of IgA nephropathy and C3 glomerulonephritis gradually increased.

The main pathological types of secondary glomerulonephritis were lupus nephritis and HSP. The differences in the male to female ratio and age distribution of these two diseases were statistically significant, with their prevalence also changing in the past nine years.

Hereditary nephropathy accounted for only a small proportion of children's kidney diseases. However, with the improvement of social living conditions and society's better understanding of these diseases, there are unlimited research prospects for studying hereditary renal disease in the future.

\section{Declarations}

\section{Ethics approval and consent to participate}

All methods in this study were performed in accordance with the relevant guidelines and regulations. The experimental protocols were approved by Ethics Committee of the First Affiliated Hospital of Zhengzhou University. As study is retrospective, Ethics Committee of the First Affiliated Hospital of Zhengzhou University approved the waiver of informed consent. This study didn't contain information or images that could lead to identification of a study participant

\section{Consent for publication}

We give our permission for this article to be published publicly in the journal BMC Pediatrics.

\section{Availability of data and materials}

All data generated or analyzed during this study are included in this published article and its supplementary information files.

\section{Competing interests}

I declare that the authors have no competing interests as defined by $\mathrm{BMC}$, or other interests that might be perceived to influence the results and/or discussion reported in this paper.

\section{Funding}

Not applicable. 


\section{Authors' contributions}

Jin Zhang wrote the main manuscript text and prepared most figures of the text, Wanyu Jia and Zhen Liu helped to collect the clinical data, Huiqin Zeng and Peipei Shi helped to design this study, Jianjiang Zhang revised and modified the manuscript $₫$ All authors reviewed the manuscript.

\section{Acknowledgements}

We would like to thank all the authors and the organizations that contributed to this article. Also, we would like to express their gratitude to EditSprings (https://www.editsprings.com/) for the expert linguistic services provided.

\section{References}

1. Pediatric Nephrology Group, Chinese Pediatric society. Renal biopsy findings in Chinese: a national cooperative study on 2315 cases. Chin J Pediatr , 1996, 34(5): 319-323.

2. Demircin G, Delibas A, Bek K, et al. A one-center experience with pediatric percutaneous renal biopsy and histopathology in Ankara, Turkey. Int Urol Nephrol. 2009;41:933-939.

3. Ding Y, Yang XQ. Pathologic analysis of 246 cases with renal biopsy in children. J appl Clin Pediatr, 2009, 24(17): 1322-1324.

4. .Kanodia KV, Vanikar AV, Nigam LK, et al. Pediatric renal biopsies in India: A single centre experience of six years. Nephrourol Mon. 2015;7:e25473-e25428.

5. Yu MC, Lee F, Huang WH, Hsueh S. Percutaneous ultrasound-guided renal biopsy in children: The need for renal biopsy in pediatric patients with persistent asymptomatic microscopic hematuria. Biomed J. 2014;37:391-397. 6.

6. Chio IH, Jeong HJ, Han DS, et al. An analysis of 2361 cases of renal biopsy in Kerea, Yonsei M J, 1991, 32(1):9-15.

7. Bazina M, Glavina-Durdov M, Scukanec-Spoljar M, et a. Epidemiology of renal disease in children in the region of southern Grotia: a 10-year review of regional renal biopsy datebases. Med Sci Monit, 2007, 13(4):172-176.

8. Mohapatra A, Kakde S, Annapandian VM, et al. Spectrum of biopsy proven renal disease in South Asian children: two decades at a tropical tertiary care centre. Nephrology(Carlton), 2018, 23(11): 1013-1022.

9. Feng LY, Jiang WM, Lan WW,et al. Pathological and clinical analysis of 977 kidney biopsies in children. Journal of Shanghai Jiaotong University, 2014, 34(03): 333-337.

10. Abdel-Hafez MA, Abdel-Nabi H, El-Camasy M, et al. Histopathological patterns of renal disease in Egyptian children: A single-center experience. Saudi J Kidney Dis Transpl, 2017, 28(5): 1085-1091. 
11. Hadidi R, Hadidi M, alDabbas M. Spectrum of biopsy-proven kidney disease in children at a Jordanian hospital. Saudi J Kidney Dis Transpl, 2014, 25(3): 680-683.

12. Fei $L J$, Hua $W$, et al. Correlation between urinary protein fraction and its pathological type in children with glomerular diseases. Chongqing Med Sci,2016,45(11): 1528-1530.

13. Manzi S. Epidemiology of lupus erythematosus. Am J Manag Care, 2011,7(16):474-479.

14. Wu TH, Li ZH, Duan CR, et al. Pathological analysis of 2887children with renal diseases. Journal of Chinese Physician, 2010,12(12): 1708-1709.

15. Kanda N, Tamaki K. Estrogen enhances immunoglobulin production by human PBMCs. J Allergy Clin Immunol, 1999, 103(2): 282-288.

16. Coppo R, Gianoglio B, Porcellini MG, et al. Frequency of renal disease and clinical indications for renal biopsy in children ( report of the Italian National Registry of Renal Biopsies in Children). Nephro Dial Transplant,1998,13(2):293-297.

17. Hou J HX, Zhou ML, et al. Changes in the Spectrum of Kidney Diseases: An Analysis of 40,759 Biopsy-Proven Cases from 2003 to 2014 in China. Kidney Dis(Basel), 2018, 4(1):10-19.

18. Yang Y, Zhang Z, Zhuo L, et al. The Spectrum of Biopsy-Proven Glomerular Disease in China: A systematic Revie. Chin Med J(Engl), 2018, 131(6):731-735.

\section{Figures}

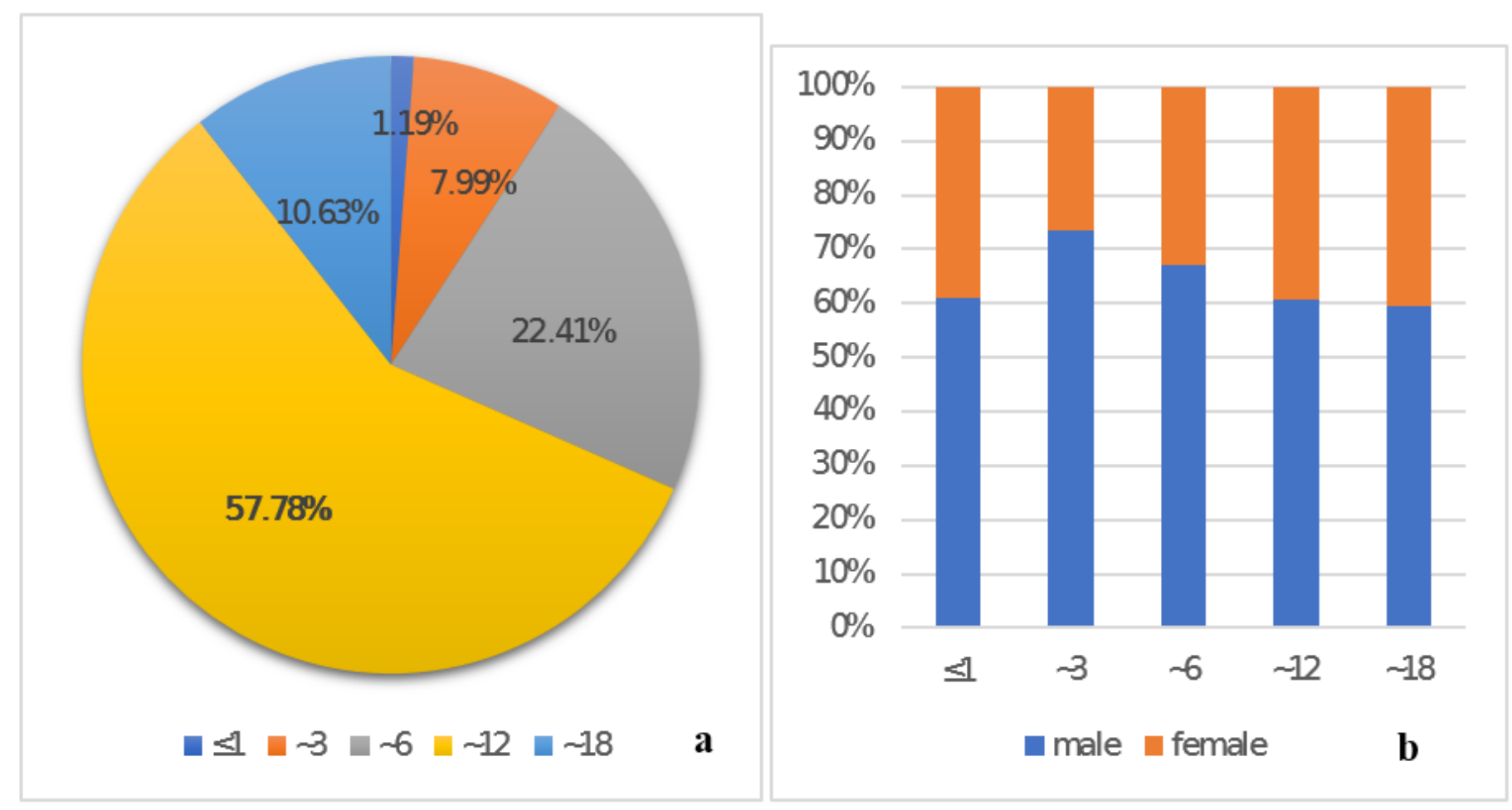

Figure 1 
The distribution of renal biopsy based on different age groups and genders. (a) The distribution of renal biopsy based on different age groups. (b) The gender distributions of renal biopsy based on age.
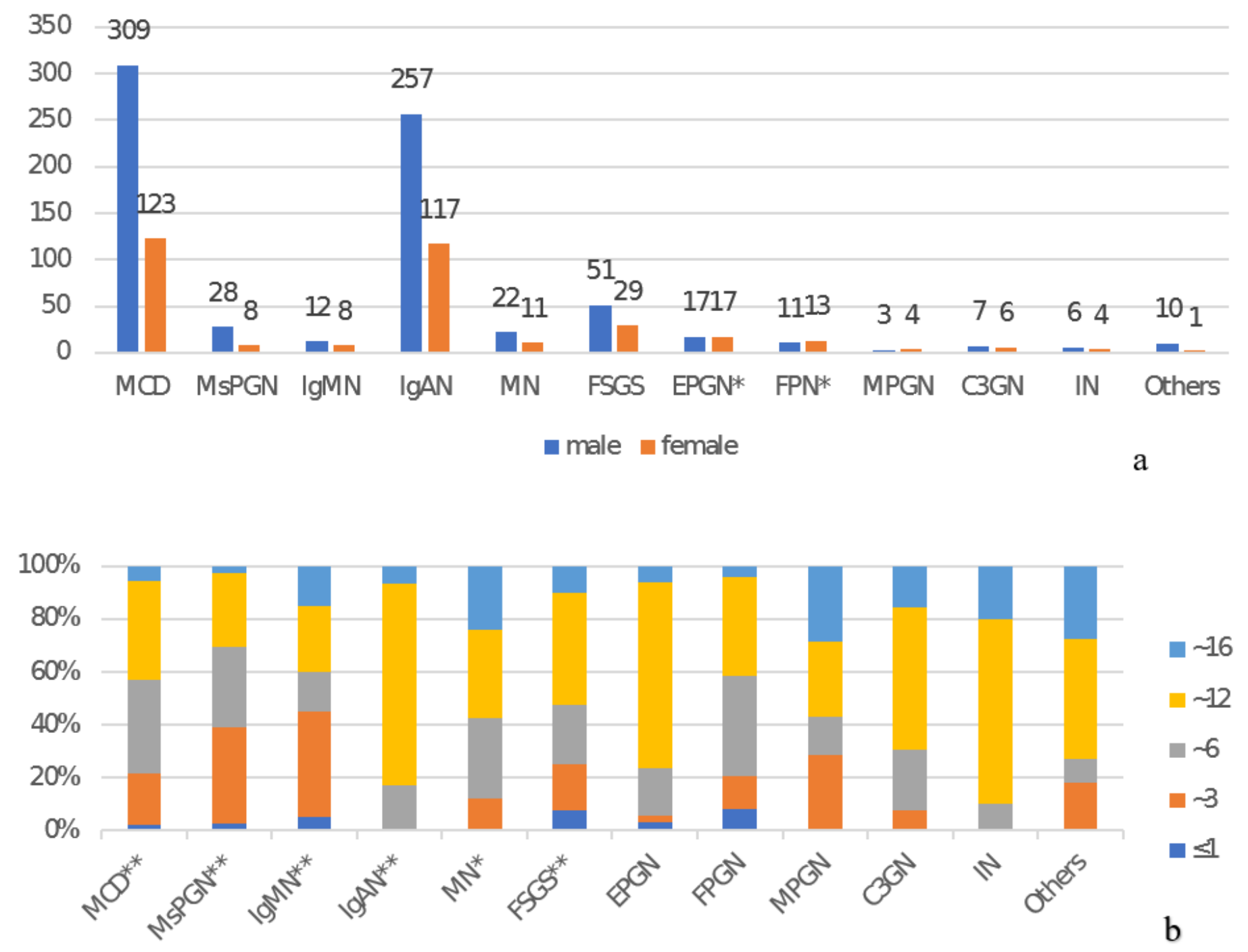

Figure 2

The gender and age distributions of primary glomerular disease according to pathological findings. (a) The gender distributions according to pathological findings. (b) The age distributions according to pathological findings. * $p \llbracket 0.05, * * p \rrbracket 0.01$. 

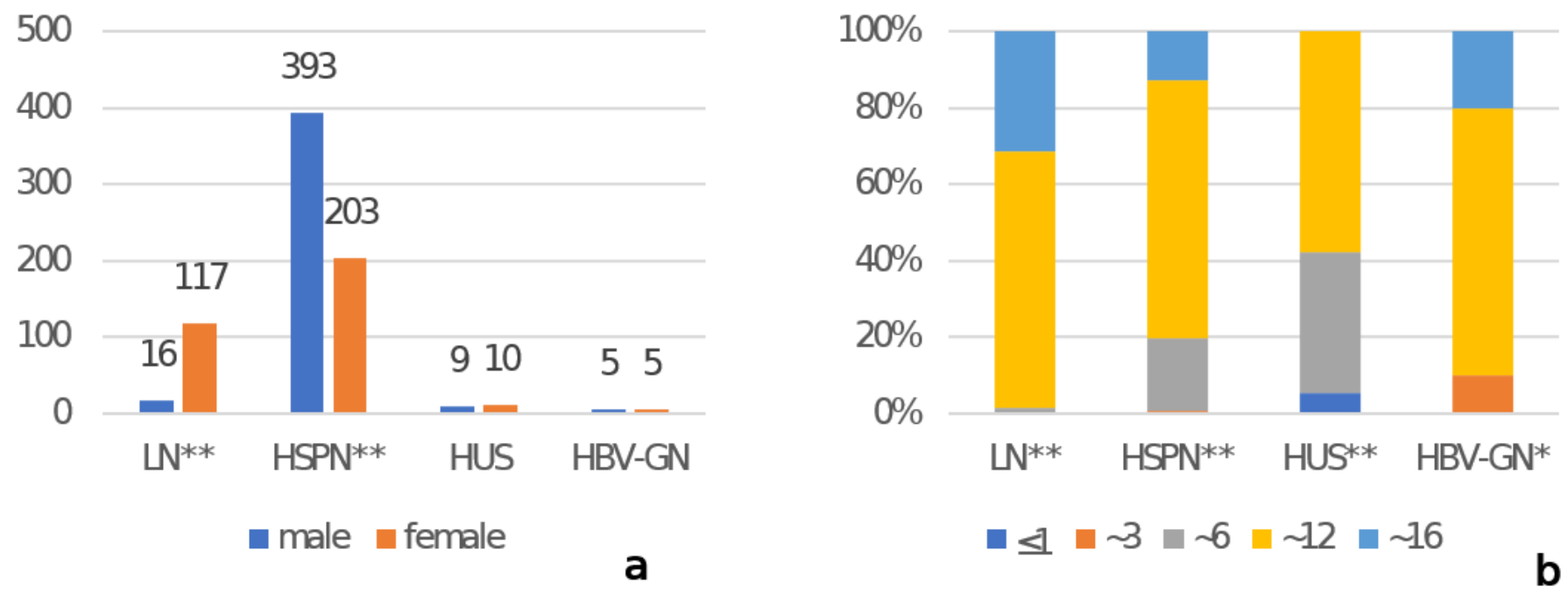

\section{Figure 3}

The gender and age distributions of secondary glomerular disease according to pathological findings. (a) The gender distributions according to pathological findings. (b) The age distributions according to pathological findings. * $p \otimes 0.05, * \star p \otimes 0.01$.

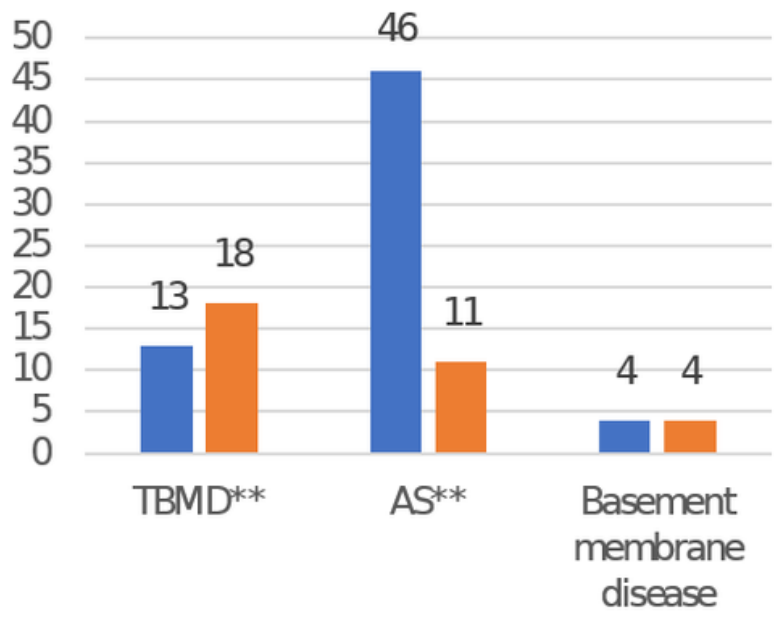

male female

a

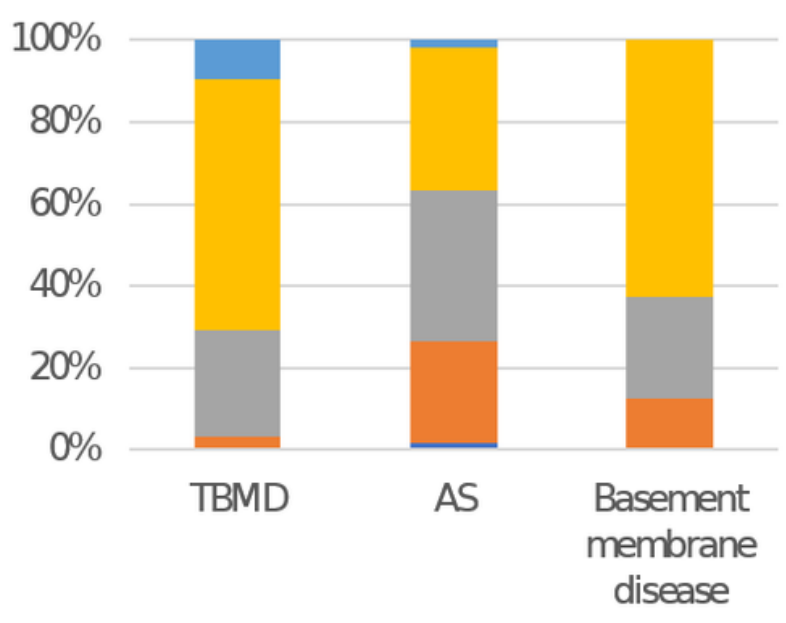

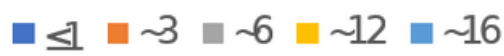

\section{Figure 4}

The gender and age distributions of hereditary glomerular disease according to pathological findings. (a) The gender distributions according to pathological findings. (b) The age distributions according to pathological findings. 

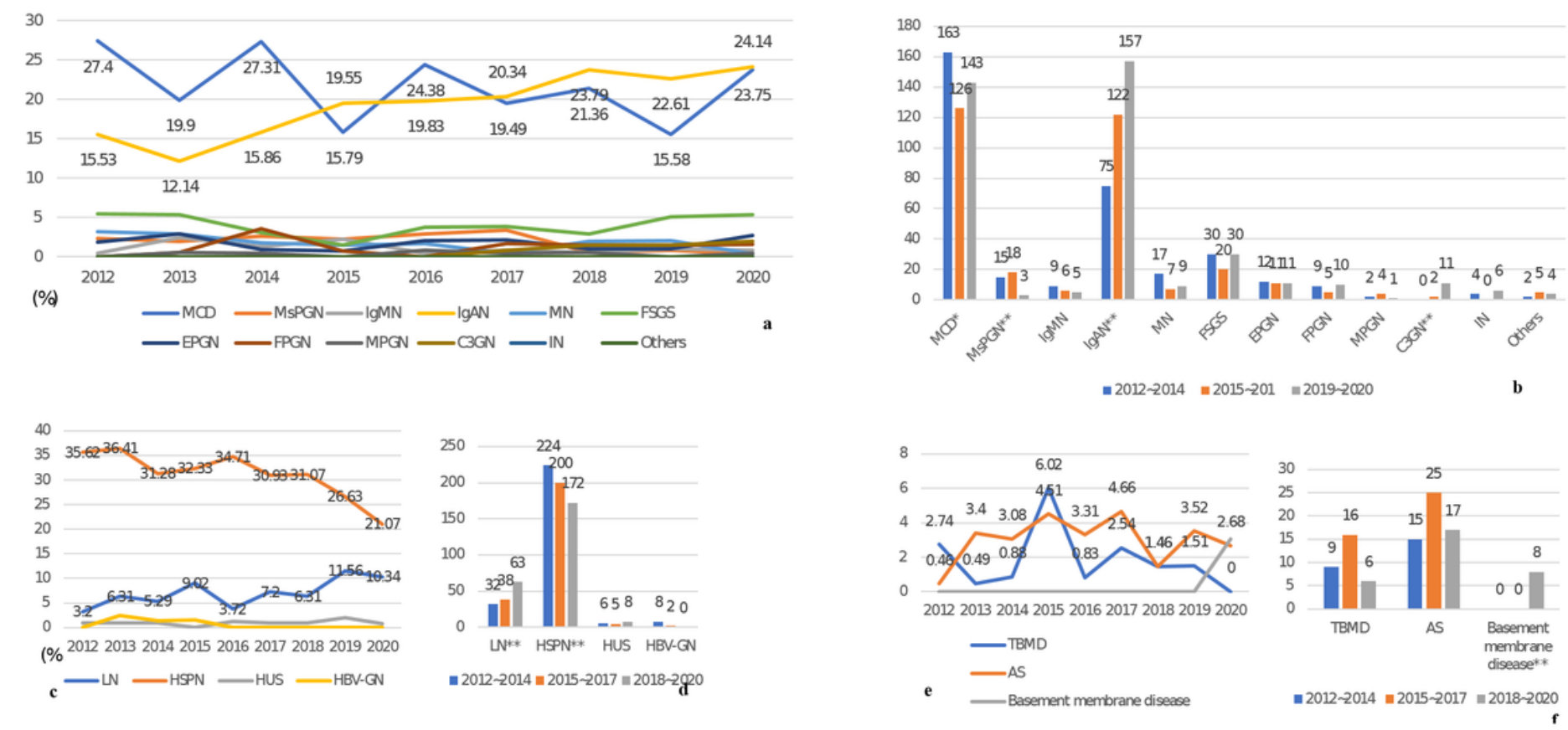

Figure 5

Annual prevalence of various pathological findings and the temporal trends in disease frequencies. (a) The annual prevalence of primary glomerular disease. (b) The changing prevalence of primary glomerular disease according to periods. (c) The annual prevalence of secondary glomerular disease. (d) The changing prevalence of secondary glomerular disease according to periods. (e) The annual prevalence of hereditary glomerular disease. (f) The changing prevalence of hereditary glomerular disease according to periods. * $p \varangle 0.05,{ }^{\star \star} p \rrbracket 0.01$. 